\title{
NOUVELL
}

\section{Le déterminisme artère-veine : la quête du GrI}

Gilles L'Allemain

activée par rayonnement laser, la même équipe peut affirmer que le devenir artériel ou veineux d'un vaisseau

Centre de Biochimie Cnrs/Inserm,

Faculté des Sciences, Parc Valrose, 06108 Nice Cedex 02, France. est prédéterminé dans le mésoderme latéral postérieur dès le stade angioblaste [5]. Une analyse par microangiographie montre que l'injection dans l'embryon

Mais comment, et à quel niveau du développement, la proportion correcte de veines et d'artères est-elle déterminée avec précision? Les vaisseaux embryonnaires des vertébrés sont dérivés de précurseurs appelés angioblastes, localisés initialement à la périphérie de l'embryon au niveau du mésoderme latéral postérieur. Les angioblastes migrent ensuite vers l'axe longitudinal central de l'embryon pour former l'aorte primitive et les premières veines. Le modèle du poisson-zèbre est très utilisé pour l'étude du développement embryonnaire car les embryons sont transparents, ce qui permet une visualisation rapide des porteurs de défauts anatomiques. Dans le domaine de la vascularisation, cet organisme est d'autant plus intéressant qu'il peut survivre en l'absence de flux sanguin. Fin 1995, un mutant présentant une altération du système cardiovasculaire fut appelé Gridlock ( $\mathrm{Grl}$ ) car dépourvu de vaisseaux principaux [2]. Ce gène Grl code pour un facteur transcriptionnel à motif HLH (helix loop helix) connu chez la drosophile parce qu'il participe au destin des précurseurs neuronaux [3]. En mars 2000, le groupe de Mark Fishman (Harvard Medical School, USA) remarqua que l'expression du gène en question coïncidait avec les régions où les angioblastes étaient détectés, dans la région mésodermique puis dans l'aorte primitive, mais pas dans les veines [4]. Aujourd'hui, à l'aide d'une sonde injectée au stade embryonnaire (1->2 cellules) et couplée à un fluorochrome dont l'émission de lumière est spécifiquement d'oligonucléotides anti-sens de Grl provoque, de façon dépendante de la dose, la rupture des structures aortiques jusqu'à leur disparition complète aux doses les plus élevées, avec une augmentation concomitante du nombre de veines. Quant à la surexpression de Grl, elle entraîne une réduction de l'arbre veineux sans affecter le nombre des artères. Ces résultats indiquent que la protéine $\mathrm{Grl}$ est requise tout le long de l'arbre artériel, et que l'arbre veineux s'étend aux dépens de ce dernier. Les auteurs proposent donc que $\mathrm{Gr}$ soit un répresseur du devenir veineux, et soit nécessaire - mais non suffisant - à la formation des artères. Comme il a été montré que la protéine ephb4 est spécifique des veines, les auteurs indiquent en outre [5] que l'ajout progressif d'anti-sens de Grl s'accompagne d'une expression croissante de ephb4 et d'une répression concomitante du marqueur des artères efnb2. Ce résultat établit ainsi le rôle primordial joué par la protéine Grl dans le déterminisme artère versus veine. Mais quelle signalisation emprunte Grl ? La protéine Grl appartient à la famille des répresseurs transcriptionnels Hrt (hairy-related transcriptional repressor), activés en aval de la voie de signalisation des récepteurs Notch. Les auteurs montrent, toujours dans la même étude [5], que l'injection du domaine intracellulaire de Notch-1, capable de mimer l'activation des récepteurs Notch par leurs ligands, induit l'expression de Gridlock (Figure I). A l'inverse, des quantités croissantes de $\mathrm{Su}(H)$, gène suppresseur de Notch, l'angioblaste. 
réduisent les doses présentes de Grl et provoquent la disparition progressive des structures aortiques au profit des structures veineuses. Cette étude lève ainsi le voile sur le rôle prépondérant de la voie Notch-1 -> Gridlock dans le déterminisme artério-veineux. II serait passionnant d'étudier à présent le rôle de ces deux protéines dans la reprogrammation cellulaire qui se produit, chez l'adulte, après le remplacement chirurgical d'une artère endommagée par la veine saphène. $\diamond$

Specification of angioblasts to artery or vein fate
1. Wang HU, Chen ZF, Anderson DJ. Molecular distinction and angiogenic interaction between embryonic arteries and veins revealed by ephrin-B2 and its receptor Eph-B4. Cell 1998; 93: 741-53.

2. Weinstein BM, Stemple DL, Driever W, Fishman MC. Gridlock, a localized heritable vascular patterning defect in the zebrafish. Nat Med 1995 ; $1: 1143-7$.

3. Artavanis-Tsakonas $S$. Rand MD, Lake RJ. Notch

\section{NOUVELLE}

\section{L'hepcidine, un contrôle essentiel de l'absorption du fer}

Gaël Nicolas, Sophie Vaulont
$>$ L'homéostasie du fer au niveau de l'organisme repose sur une régulation fine de l'absorption intestinale du fer alimentaire (pour revue, voir [1]). Cette absorption se fait par les entérocytes mûrs situés au sommet de la villosité duodénale et met en jeu un grand nombre de protéines incluant des réduc-

$(\rightarrow) \mathrm{m} / \mathrm{s}$ 2000, n6-7, des transporteurs memp. 833 branaires $(\rightarrow)$. L'expression intégrée et coordonnée de ces différentes protéines dépend de signaux envoyés par l'organisme qui varient en fonction du niveau des réserves tissulaires en fer (on parle du signal store regulator), ou de l'activité érythropoïétique de la moelle osseuse (on parle du signal erythroid regulator). Ce sont les précurseurs situés dans la crypte de la villosité intestinale qui reçoivent ces signaux et qui, en se différenciant le long de la villosité en entérocytes mûrs, permettront le contrôle de l'absorption de fer. Ainsi, dans des conditions physiologiques nor- males, les entérocytes sont-ils programmés pour s'adapter à la demande en fer de l'organisme. La nature des signaux permettant la programmation des entérocytes a longtemps été recherchée et il semble qu'une des molécules participant à cette programmation ait été récemment identifiée. II s'agit de l'hepcidine, une protéine synthétisée dans le foie sous la forme d'un pré-propeptide et excrétée dans la circulation sous la forme d'un peptide mûr de 25 acides aminés. Le peptide circulant a été purifié indépendamment par deux groupes sur la base de ses propriétés anti-microbiennes [2, 3].

La découverte de l'implication de l'hepcidine dans le métabolisme du fer repose sur une observation inattendue de notre laboratoire faite dans un modèle de souris déficientes (souris knock-out) pour le facteur de transcription USF2 (upstream stimulatory factor) [4]. Dès l'âge de trois mois, le foie et le pancréas de ces souris sont de couleur marron, suggérant leur surcharge en fer. signaling: cell fate control and signal integration in development. Science 1999 ; 284 : 770-6.

4. Zhong TP, Rosenberg $M$, Mohideen MA, Weinstein B Fishman MC. Gridlock, an $H L H$ gene required for assembly of the aorta in zebrafish. Science 2000 ; $10 ; 287: 1820-4$

5. Zhong TP, Childs S, Leu JP, Fishman MC. Gridlock signalling pathway fashions the first embryonic artery. Nature 2001 ; 414 : 216-20.

Département de génétique, développement et pathologie moléculaire, Institut Cochin, Inserm, Cnrs et Université René Descartes, Faculté de Médecine Cochin-Port Royal, 24, rue du Faubourg Saint-Jacques, 75014 Paris, France.

De fait, les souris Usf2-/- développent une surcharge martiale avec un tableau clinique en tout point similaire à celui des sujets atteints d'hémochromatose. L'hémochromatose est une maladie génétique à caractère autosomique récessif liée, dans $80 \%$ des cas, à l'existence d'une mutation (C282Y) du gène $\mathrm{Hfe}-\mathrm{l}(\rightarrow)$. Cette affection se caractérise cliniquement par une $\rightarrow) \mathrm{m} / \mathrm{s}$ $1998, n^{\circ} 12$, p. 1387 hyper-absorption digestive de fer qui aboutit à son accumulation dans les tissus. Curieusement, les macrophages de la rate échappent à ce processus d'accumulation du fer. Si aujourd'hui le rôle de la protéine HFE n'est pas entièrement élucidé, on pense qu'une de ses fonctions serait d'opérer une régulation négative de la capture du fer via le complexe transferrine/récepteur de la transferrine. Les souris Usf2 $2^{-/-}$ miment parfaitement le phénotype du 